\title{
PRODUCTIVIDAD DE HÍBRIDOS VARIETALES DE MAÍZ DE GRANO AMARILLO PARA VALLES ALTOS DE MÉXICO ${ }^{1}$
}

\author{
Margarita Tadeo-Robledo², Alejandro Espinosa-Calderón ${ }^{3}$, Raymundo Guzmán-Máximo², \\ Antonio Turrent-Fernández $z^{3}$,Job Zaragoza-Esparza ${ }^{2}$, Juan Virgen-Vargas ${ }^{3}$
}

\begin{abstract}
RESUMEN
Productividad de híbridos varietales de maíz de grano amarillo para valles altos de México. El objetivo de este trabajo fue evaluar la productividad de híbridos varietales no convencionales de grano amarillo con respecto a las variedades progenitoras. En el Instituto Nacional de Investigaciones Forestales, Agrícolas y Pecuarias (INIFAP) y la Facultad de Estudios Superiores Cuautitlan, de la Universidad Nacional Autónoma de México (FESC-UNAM), se generaron variedades precoces de grano amarillo, con ellas, en el año 2010, se llevaron a cabo combinaciones con dos cruzas simples. Los genotipos fueron evaluados en 2012, se realizaron tres experimentos uniformes, con quince genotipos y tres repeticiones, estableciéndose dos ensayos en la FESC-UNAM, y uno en el Campo Experimental Valle de México. El análisis combinado detectó diferencias altamente significativas para la variable rendimiento en genotipos, ambientes y la interacción genotipos $\mathrm{x}$ ambiente. La mejor combinación no convencional de cruza simple x variedad, CS156 x V 54 A (6796 kg/ha), superó en $38 \%$ el rendimiento de V-54 A (4918 kg/ha) y la segunda mejor combinación (CS156 x V 55 A), rindió 6204 $\mathrm{kg} / \mathrm{ha}$, que fue superior en $17,3 \%$ con respecto a su variedad progenitora V-55 A (5288 kg/ha). Se incrementó el rendimiento al cruzar las variedades mejoradas de grano amarillo con cruzas simples, lo que representa una alternativa para ofrecer genotipos con mayor capacidad productiva manteniendo ciclo vegetativo y con facilidad para el abastecimiento de semilla de productores de maíz.
\end{abstract}

Palabras claves: Zea mays L., rendimiento de maíz, variedades no convencionales.

\begin{abstract}
Productivity of intervarietal hybrids of yellow grain corn for high valleys of México. The objective of this study was to evaluate the productivity of non-conventional intervarietal maize hybrids with yellow endosperm grain with respect to their parental varieties. At the National Institute for Forestry, Agricultural and livestock Research (INIFAP) and the Faculty of Superior Studios Cuautitlán, of the National Autonomous University of Mexico (FESC-UNAM), early maize varieties of yellow grain were generated. In the year 2010, combinations with two single crosses were carried out. In 2012, 15 genotypes were evaluated in three uniform experiments, with three repetitions. Two trials were planted in the FESC-UNAM, and the other one in the Experimental Station of Valley of Mexico. The combined analysis detected differences highly significant to the variable performance in genotypes, environments and the interaction genotype $\mathrm{x}$ environment. The best non-conventional combination of single cross x variety, CS156 x V 54 A $(6,796 \mathrm{~kg} / \mathrm{ha})$, yielded $38 \%$ more than V-54 A (4918 kg/ha) and the second best combination (CS156 x V 55), yielded 6,204 kg/ ha, which was superior by $17.3 \%$ compared to its progenitor variety V-55 A $(5,288 \mathrm{~kg} / \mathrm{ha})$. The grain yield was increased by crossing the improved varieties with the single crosses. This represents an alternative to offer genotypes with greater productive capacity while maintaining its same maturity and with ease for the supply of maize seed for the farmers.
\end{abstract}

Keywords: Zea mays L., maize yield, non-conventional varieties.

1 Recibido: 1 de marzo, 2014. Aceptado: 6 de setiembre, 2014. Investigación realizada en el programa UNAM-DGAPA, en el proyecto PAPIIT: IT201312-3. México.

2 Universidad Nacional Autónoma de México (UNAM), Facultad de Estudios Superiores Cuautitlán (FESC). México. Carr. Cuautitlán - Teoloyucán, Km 2.5. Cuautitlán Izcalli, Estado de México. C.P. 54714. tadeorobledo@yahoo.com, chaktum@gmail.com, jobzaragoza4920@ yahoo.com

3 Instituto Nacional de Investigaciones Forestales, Agrícolas y Pecuarias (INIFAP), Campo Experimental Valle de México (CEVAMEX). México, Carr. Los Reyes-Texcoco, Km 13.5, CP. 56250. espinoale@yahoo.com.mx (autor para correspondencia), aturrent37@yahoo.com. mx, jvirgen_vargas@hotmail.com 


\section{INTRODUCCIÓN}

México requiere 32,5 millones de toneladas de grano de maíz al año para cubrir su demanda aparente. Dado que se producen 22,5 millones de toneladas de maíz de grano blanco, cada año se importan diez millones de toneladas de maíz de grano amarillo, por lo que se requiere incrementar la producción de maíz (Turrent, 1994; Turrent, 2009; Espinosa et al., 2013). De la superficie cultivada nacional de maíz, 1,5 millones de hectáreas se ubican en altitudes de 2200 a $2600 \mathrm{msnm}$, en los Valles Altos de la Mesa Central. De esta extensión, 800 mil hectáreas se cultivan en temporal estricto, el cual generalmente se presenta en forma tardía, lo que limita la fecha de siembra y por ello la productividad del cultivo es limitada $(1,2$ t/ha), por el temporal deficiente y fecha de siembra tardía, que frecuentemente coincide con la incidencia de heladas tempranas (Ávila et al., 2009; Espinosa et al., 2010).

De la industrialización del grano amarillo, se derivan: almidón, glucosa, alta fructosa, gluten, fibra, sorbitol (jarabe), aceites, maltodextrinas, color caramelo, dextrosa, proteínas y sus aplicaciones, lo que propicia que se requieran grandes volúmenes de grano de maíz amarillo, ahora de 10 millones de toneladas y que podrían ser mayores en un futuro, con la fuerte demanda para el uso de maíz en la elaboración de etanol en los EE. UU. (Espinosa et al., 2010; Espinosa et al., 2011). Por lo mencionado anteriormente, el uso de semilla mejorada es un elemento clave en muchos países en desarrollo, para alcanzar niveles competitivos en la producción (Espinosa et al., 2008b; Tadeo et al., 2012).

Una alternativa para incrementar la producción y atender la demanda de grano de maíz amarillo es el uso de variedades mejoradas de grano amarillo de ciclo corto, que aprovechen las condiciones agroclimáticas disponibles; para ello, se desarrollaron en la Facultad de Estudios Superiores Cuautitlán, de la Universidad Nacional Autónoma de México (FESC, UNAM), algunas variedades de maíz de grano amarillo, de ciclo precoz, como Oro Ultra 3C, Oro Ultra 2C, Oro Plus 2D (Tadeo y Espinosa, 2004; Tadeo et al., 2012). En forma similar, en el Instituto Nacional de Investigaciones Forestales, Agrícolas y Pecuarias (INIFAP), en los últimos años se ha promovido tres variedades de grano amarillo de ciclo precoz denominadas V-53 A, V-54 A,
V-55 A (Espinosa et al., 2008b; Espinosa et al., 2010; Espinosa et al., 2011).

Una posibilidad para elevar el rendimiento de estas variedades de la FESC y el INIFAP, es la obtención de híbridos no convencionales, los cuales se definen como híbridos obtenidos a partir de la combinación de progenitores que no cumplen con la conformación clásica, es decir, que no son híbridos simples, trilineales o dobles. Estos híbridos no convencionales son resultado de la combinación de una variedad x un híbrido, una variedad x una línea. Generalmente presentan facilidad para la producción de semillas, así como rendimiento favorable, que es con frecuencia superior a ambos progenitores (Espinosa et al., 1999). Con base en lo anterior, durante el ciclo primavera verano 2010, se efectuaron cruzamientos de algunas de estas variedades de grano amarillo con dos cruzas simples, obteniéndose algunos híbridos no convencionales. Con la semilla obtenida, en el ciclo primavera verano 2012, se evaluaron algunos de estos híbridos de grano amarillo con respecto a las variedades progenitoras.

El objetivo de este trabajo fue evaluar la productividad de híbridos varietales no convencionales de grano amarillo con respecto a las variedades progenitoras.

\section{MATERIALES Y MÉTODOS}

La primera etapa de la investigación se llevó a cabo en el ciclo primavera verano 2010, en el cual se efectuaron polinizaciones manuales, es decir, cruzamientos para obtener semilla de combinaciones de híbridos no convencionales de maíz, en donde se emplearon tres variedades de maíz de polinización libre del INIFAP, V-53A, V-54 A y V-55 A, las dos últimas ya inscritas en el Catálogo Nacional de Variedades Vegetales (CNVV), por el INIFAP y por lo tanto, en uso comercial (Espinosa et al., 2008a; Espinosa et al., 2008b; Espinosa et al., 2010; Espinosa et al., 2011) y también tres variedades de la FESCUAM: Oro Ultra 2C, Oro Ultra $3 \mathrm{C}$ y Oro Plus 2D. Variedades que se recomiendan en siembras retrasadas de mayo y hasta junio, ya que son precoces. Estas seis variedades fueron sembradas en el ciclo primavera verano 2010, y en la etapa de floración, se cubrieron sus jilotes, mismos que se emplearon para llevar a cabo cruzas manuales de ellas con plantas de las 
cruzas simples de grano amarillo denominadas CS156 y CS155. De esta manera, se obtuvieron cinco híbridos no convencionales (CS156 X V-54 A, CS156 X V-55 A, CS156 X OU2C, CS156 X OU3C, CS156 X OP2D), dos híbridos no convencionales más CS155 X V 54 A y CS155 X V 53 A, y se obtuvo semilla de las cruzas simples (CS156 y CS155) y de las variedades (V 53 A, V 54 A, V-55 A, OU2C, OU3C, OP2D). Con la semilla obtenida (siete híbridos no convencionales, dos cruzas simples y seis variedades de polinización libre), se llevó a cabo la segunda etapa del trabajo, en el ciclo primavera verano 2012, en el cual se establecieron tres experimentos uniformes como se describe en este artículo.

Se establecieron tres experimentos, uno en el Campo Experimental Valle de México (CEVAMEX) dependiente del Instituto Nacional de Investigaciones Forestales, Agrícolas y Pecuarias (INIFAP), ubicado en Santa Lucía de Prias, Coatlinchán, Municipio de Texcoco, Estado de México en el municipio de Texcoco, Estado de México, a una altitud de $240 \mathrm{~m}$, la fecha de siembra fue el 27 de mayo de 2012. Otros dos experimentos se establecieron, con diferencia en fecha de siembra, uno el 17 de mayo de 2012 y otro el 01 de junio de 2012, en el Rancho Almaraz de la Facultad de Estudios Superiores Cuautitlán, Campo 4, de la Universidad Nacional Autónoma de México (UNAM), ubicado en los $99^{\circ} 11^{\prime} 42^{\prime \prime}$ LW y $19^{\circ} 41^{\prime}$ 35 " LN, y una altitud de $2274 \mathrm{msnm}$. De acuerdo a la clasificación de Köpen modificada por García (2004), el clima de Cuautitlán se clasifica como C(W0)(W)b (i"). La precipitación anual promedio histórico fue de 609,2 mm (García, 2004).

En cada uno de los tres experimentos se evaluaron quince genotipos, entre los cuales se incluyeron las variedades (6), cruzas simples (2) y combinaciones (7) de estas variedades por las cruzas simples (híbridos no convencionales). En los tres experimentos la densidad de población fue de 45000 plantas/ha, recomendada para este tipo de siembras retrasadas y de limitada humedad en la siembra (Espinosa et al., 2010), la parcela experimental constó de un surco de $5 \mathrm{~m}$ de largo por $80 \mathrm{~cm}$ de ancho, dando un total de $4 \mathrm{~m}^{2}$ como parcela útil. Se utilizó un diseño de bloques completos al azar, con tres repeticiones. En los tres casos, los experimentos se manejaron bajo condiciones de secano, humedad que procede de la precipitación pluvial, en los tres casos las siembras a fines de mayo y junio se consideran fechas de siembra retrasadas.
El manejo agronómico de los experimentos se llevó a cabo aplicando el tratamiento fertilizante de 80-40-00, al momento de hacer el surcado, esta dosis de fertilizante es recomendada para condiciones de fecha de siembra y secano, para lo cual se emplearon como fuentes: nitrato de amonio para nitrógeno y superfosfato de calcio triple para fósforo. Para el control de malezas se aplicó herbicida en dos ocasiones: la primera, un día después de la siembra, con la mezcla de $1 \mathrm{~L}$ de 2,4 D Amina y $2 \mathrm{~kg}$ de Atrazina, por hectárea; la segunda aplicación fue 20 días después de la siembra, con una mezcla de $1 \mathrm{~L}$ de Nicosulfuron más 0,51 de 2,4 D Amina y $2 \mathrm{~kg}$ de Atrazina, por hectárea. La preparación del terreno se realizó de forma mecánica y consistió en barbecho, rastreo, cruza y surcado a $80 \mathrm{~cm}$.

La siembra en fechas retrasadas (segunda quincena de mayo y 1 de junio) se depositaron en los tres experimentos dos semillas por cada $50 \mathrm{~cm}$, posteriormente se raleó, para obtener la densidad de población recomendada de 45000 plantas/ha, bajo condiciones de secano, en siembras retrasadas. La cosecha se realizó de forma manual en la segunda quincena de noviembre de 2012, en los tres experimentos.

Se evaluaron las variables: floración masculina (días), cuando el 50\% de las plantas de la parcela liberaban polen, floración femenina (días), cuando el $50 \%$ de las plantas, en la parcela habían expuesto los estigmas, en por lo menos $3 \mathrm{~cm}$, altura de planta $(\mathrm{cm})$, tomada en cinco plantas de la base del tallo al nudo de inserción de la espiga; altura de mazorca $(\mathrm{cm})$, de la base del tallo al nudo de inserción de la mazorca superior. Los datos de las variables hileras por mazorca, longitud de mazorca $(\mathrm{cm})$, granos por hilera, se tomaron de cinco mazorcas, obteniéndose al final un promedio, en forma similar; es decir, en las cinco mazorcas se multiplicó hileras por mazorca por granos por hilera, para obtener número de granos por mazorca. Los datos correspondientes a humedad de grano (\%), fueron obtenidos a través de un determinador de humedad eléctrico tipo Stenlite, en el caso del porcentaje de grano/olote, se obtuvo al desgranar cinco mazorcas recién cosechadas y definir el cociente de peso de grano entre peso de grano más olotes; se tomó el peso de 200 granos (g), los datos de las diversas variables fueron analizados considerando los factores de variación ambiente, genotipos, así como la interacción ambiente $\mathrm{x}$ genotipos, con base en el Statistical Analysis Systems (SAS Institute, 1996). 
Para calcular el rendimiento de grano se aplicó la fórmula siguiente (Espinosa et al., 2010):

Rendimiento $=(\mathrm{PC} \times \% \mathrm{MS} \times \% \mathrm{G} \times \mathrm{FC}) / 8600$

en donde:

$\mathrm{PC}=$ peso de campo de la totalidad de las mazorcas cosechadas por parcela expresada en kilogramos.

$\% \mathrm{MS}=$ por ciento de materia seca de la muestra de grano de cinco mazorcas recién cosechadas.

$\% \mathrm{G}=$ por ciento de grano.

$\mathrm{FC}=$ Factor de conversión para obtener el rendimiento por ha que se obtiene al dividir 10000 $\mathrm{m}^{2} / \mathrm{el}$ tamaño de la parcela útil en $\mathrm{m}^{2}$.

$8600=$ valor constante que permite estimar el rendimiento con una humedad al $14 \%$, que se usa para el grano en forma comercial.

El diseño de campo fue en bloques completos al azar, con tres repeticiones. Para el análisis estadístico de los datos de las diversas variables, se utilizó el Statistical Analysis Systems (SAS Institute, 1996). Este se realizó combinando los tres ambientes, considerando como fuentes de variación el ambiente, los genotipos y la interacción genotipos x ambientes. La comparación de medias se hizo con la prueba de Tukey a 0,05 de probabilidad de error $(p \leq 0,05)$.

Se considera a variedades precoces cuando exhiben floración masculina de 67 a 77 días, variedades intermedias de 78 a 86 días a floración masculina y variedades de ciclo tardío de 87 a más de 100 días a floración masculina (Espinosa et al., 2010; Espinosa et al., 2011).

\section{RESULTADOS Y DISCUSIÓN}

En el análisis de varianza combinado de los tres experimentos para rendimiento, se detectó diferencias altamente significativas para ambientes, genotipos y la interacción ambientes x genotipos, lo cual señala que existió una respuesta diferencial de los genotipos en los ambientes de evaluación utilizados en el estudio, lo que es explicable ya que aun cuando entre las localidades se ubican en una altitud similar, al conducirse los tres ambientes bajo condiciones de secano, en combinación con características de suelo y fecha de siembra, propiciaron que se presente la respuesta diferencial de los genotipos evaluados
(Espinosa et al., 2008a; Espinosa et al., 2008b; Espinosa et al., 2010; Espinosa et al., 2011).

La media del rendimiento de los tres ambientes fue de $4663 \mathrm{~kg} / \mathrm{ha}$ y el coeficiente de variación $17,1 \%$. Para el factor de variación ambientes, se detectaron diferencias altamente significativas para floración masculina y femenina, altura de planta y de mazorca, peso de 200 granos, granos/hilera y porcentaje de materia seca; en cambio la variable longitud de mazorca presentó diferencias estadísticas significativas al 5\% de probabilidad, para el caso de la variable hileras por mazorca; no se detectaron diferencias (Cuadro 1).

En el factor de variación genotipos se detectaron diferencias altamente significativas $(\mathrm{p} \leq 0,01)$; para floración femenina, altura de planta y mazorca, peso de 200 granos, longitud de mazorca, hileras por mazorca, granos por hilera, por mazorca y diferencias significativas al nivel de $\mathrm{p} \leq 0,05$ para la variable floración masculina. Para el factor de variación genotipos $\mathrm{x}$ ambientes, floración femenina, y la variable rendimiento presentaron diferencias significativas $(\mathrm{p} \leq 0,05)$ (Cuadro 1$)$.

En la comparación de medias para ambientes, el mejor rendimiento medio, correspondió al ambiente de la FESC-UNAM 1 (17 de mayo de 2012), con $5094 \mathrm{~kg} / \mathrm{ha}$, estadísticamente superior al experimento establecido en el CEVAMEX y también al establecido en la FESC-UNAM 2, en la segunda fecha de siembra (1 de junio de 2012). El menor rendimiento medio de FESC-UNAM, podría deberse a la siembra tardía (quince días de retraso), propiciando menor número de días para completar el ciclo y probablemente afectó la productividad. La similitud de los ambientes CEVAMEX y FESC-UNAM2, podría estar influenciada por la fecha de siembra, ya que fue muy cercana una de otra, ubicándose ambas localidades a una altitud similar sobre el nivel del mar (Cuadro 2).

En las localidades de evaluación se confirmó la precocidad de las variedades, ya que los valores obtenidos de variables como floración masculina y femenina fue verificada obteniéndose respuestas similares a lo que se señala en trabajos previos (Espinosa et al., 2008b; Espinosa et al., 2010; Espinosa et al., 2011), la fecha de siembra tardía, presentó valores mayores en días a floración masculina y femenina, lo que pudo deberse a condiciones diferentes al sembrarse quince días después de la primera fecha. El menor rendimiento obtenido en el experimento de 
Cuadro 1. Cuadrados medios y significancia estadística para rendimiento ( $\mathrm{kg} / \mathrm{ha}$ ) y variables evaluadas en variedades y combinaciones con dos cruzas simples de maíz amarillo. Facultad de Estudios Superiores Cuautitlán, Universidad Nacional Autónoma de México (FESC-UNAM), México. Ciclo primavera-verano 2012.

\begin{tabular}{lccccc}
\hline Variable & $\begin{array}{c}\text { Ambiente } \\
\text { C.M. }\end{array}$ & $\begin{array}{c}\text { Genotipo } \\
\text { C.M. }\end{array}$ & $\begin{array}{c}\text { Amb. X Gen. } \\
\text { C.M. }\end{array}$ & C.V. (\%) & Media \\
\hline Rendimiento & $7204864,4^{* *}$ & $20869502,7^{* *}$ & $3549133,0^{* *}$ & 17,1 & 4663 \\
DFM & $650,78^{* *}$ & $65,53^{*}$ & 22,87 & 6,5 & 72 \\
DFF & $653,65^{* *}$ & $51,1^{* *}$ & $5,13^{*}$ & 2,1 & 74 \\
AP $(\mathrm{cm})$ & $20714,97^{* *}$ & $1380,10^{* *}$ & 353,21 & 6,9 & 218 \\
AM $(\mathrm{cm})$ & $6075,76^{* *}$ & $503,56^{* *}$ & 135,50 & 9,0 & 96 \\
PV $(\mathrm{kg} / \mathrm{hl})$ & 2012,45 & 1045,94 & 538,12 & 2,9 & 769 \\
P200G $(\mathrm{g})$ & $2164,45^{* *}$ & $170,88^{* *}$ & 30,00 & 9,2 & 61,1 \\
LM $(\mathrm{cm})$ & $9,38^{*}$ & $10,56^{* *}$ & 1,36 & 8,8 & 15,7 \\
HM & 5,89 & $9,36^{* *}$ & 1,84 & 7,9 & 15,1 \\
GH & $60,58^{* *}$ & $43,85^{* *}$ & 7,50 & 8,7 & 30,3 \\
GM & 1928,56 & $26990,20^{* *}$ & 3556,27 & 11,62 & 461 \\
$\%$ MS & $1631,82^{* *}$ & 9,53 & 355,32 & 3,8 & 83,76 \\
\hline
\end{tabular}

* = Significancia de los tratamientos al 0,05 de probabilidad. ** = Significancia de los tratamientos al 0,01 de probabilidad. DFM: días a floración masculina; DFF: días a floración femenina; AP: altura de planta; AM: altura de mazorca; PV: peso volumétrico; P200S: peso de 200 semillas; LM: longitud de mazorca; HM: hileras por mazorca; GH: granos por hilera; GM: granos por mazorca; MS: materia seca.

FESC-UNAM 2, se debió probablemente a la siembra tardía; sin embargo, aún cuando el rendimiento medio fue bajo $(4304 \mathrm{~kg} / \mathrm{ha})$, las variedades completaron su ciclo en esa fecha tardía, lo que se debe a la precocidad de los genotipos. En este caso la precocidad es una ventaja importante para lograr la culminación del ciclo y una cosecha aceptable, la cual contrasta con los rendimientos que se alcanzan en este tipo de siembras $(1,2 \mathrm{t} / \mathrm{ha})$, lo que representa una ventaja con respecto a otros materiales liberados previamente y ahora sin uso comercial como V-26 A y Amarillo Zanahoria (Espinosa et al., 2010).

Cuadro 2. Comparación de medias (Tukey) de ambientes para variables evaluadas, considerando la media de genotipos de maíz, variedades, cruzas simples e híbridos no convencionales. México. Ciclo primavera-verano 2012.

\begin{tabular}{|c|c|c|c|c|c|c|c|c|c|c|c|}
\hline Localidades & $\begin{array}{l}\text { Rend. } \\
\text { (kg/ha) }\end{array}$ & $\begin{array}{l}\text { DFM } \\
\text { (días) }\end{array}$ & $\begin{array}{c}\text { DFF } \\
\text { (días) }\end{array}$ & $\begin{array}{c}\text { AP } \\
(\mathbf{c m})\end{array}$ & $\begin{array}{l}\text { AM } \\
(\mathbf{c m})\end{array}$ & $\begin{array}{c}\text { PV } \\
(\mathrm{kg} / \mathrm{hl})\end{array}$ & $\begin{array}{c}\text { P200S } \\
(\mathrm{g})\end{array}$ & $\begin{array}{l}\text { LM } \\
(\mathbf{c m})\end{array}$ & HM & GH & GM \\
\hline FESC-UNAM 1 & $5094 \mathrm{a}$ & $72 \mathrm{~b}$ & $74 \mathrm{~b}$ & $220 \mathrm{~b}$ & $107 \mathrm{a}$ & $775 \mathrm{a}$ & $67,5 \mathrm{a}$ & $16,0 \mathrm{a}$ & $15 \mathrm{a}$ & $29 \mathrm{~b}$ & $455 \mathrm{a}$ \\
\hline CEVAMEX & $4589 \mathrm{~b}$ & $69 c$ & $70 \mathrm{c}$ & $239 a$ & $98 \mathrm{~b}$ & $770 \mathrm{ab}$ & $53,8 \mathrm{c}$ & $15,1 \mathrm{~b}$ & $15 \mathrm{a}$ & $32 \mathrm{a}$ & $468 \mathrm{a}$ \\
\hline FESC-UNAM 2 & $4304 \mathrm{~b}$ & $76 \mathrm{a}$ & $78 \mathrm{a}$ & $196 \mathrm{c}$ & $84 \mathrm{c}$ & $762 \mathrm{~b}$ & $61,9 \mathrm{~b}$ & $15,9 \mathrm{a}$ & $15 \mathrm{a}$ & $30 \mathrm{~b}$ & $461 \mathrm{a}$ \\
\hline D.S.H. $(0,05)$ & 402 & 2 & 1 & 8 & 4 & 11 & 2,8 & 0,7 & 1 & 1 & 27 \\
\hline
\end{tabular}

FESC-UNAM 1: Fecha de siembra 17 mayo 2012; CEVAMEX: Fecha de siembra 27 de mayo de 2012; FESC-UNAM 2: Fecha de siembra 1 de junio de 2012; D.S.H.: diferencia significativa honesta (corresponde a la diferencia mínima significativa de la Prueba de Tukey). DFM: días a floración masculina; DFF: días a floración femenina; AP: altura de planta; AM: altura de mazorca; PV: peso volumétrico; P200S: peso de 200 semillas; LM: longitud de mazorca; HM: hileras por mazorca; GH: granos por hilera.

Las medias con la misma letra estadísticamente en la misma columna, no tienen diferencia significativa (Tukey al 0,05 de significancia). 
En los experimentos que se establecieron en la FESC-UNAM, hubo una diferencia en la fecha de siembra con quince días entre el establecimiento de un experimento y otro. Esta diferencia, propició finalmente que la media de producción sea mayor en el ensayo de la primera siembra con una diferencia de $793 \mathrm{~kg} / \mathrm{ha}$, con respecto a la media obtenida en el ensayo de la segunda siembra, lo anterior significa que sembrar en la segunda quincena de mayo (17 de mayo), en promedio la producción fue $18,3 \%$ superior a la siembra del 1 de junio. Las variedades V-54 A, V-55 A, Oro Ultra 3 C y Oro Ultra 2C, pueden aprovecharse en este tipo de siembras, ya que en trabajos previos mostraron rendimientos y ventajas superiores con respecto a Amarillo Zanahoria (Espinosa et al., 2010; Espinosa et al., 2013).

En la comparación de medias para genotipos, para rendimiento se registraron cuatro grupos de significancia, en los tres primeros lugares se ubicaron las combinaciones CS156 x V 54 A $(6796 \mathrm{~kg} / \mathrm{ha})$, CS156 x V 55 A (6204 kg/ha), CS156 x OU2C (5985 $\mathrm{kg} / \mathrm{ha}$ ), en los dos primeros híbridos no convencionales, participaron como progenitoras las variedades V $54 \mathrm{~A}$ y V 55 A que procedieron de INIFAP (Espinosa et al., 2010; Espinosa et al., 2011) y Oro Ultra 2 C que procede de la FESC-UNAM (Tadeo et al., 2010). La variedad V 53 A $(5,947 \mathrm{~kg} / \mathrm{ha})$ exhibió rendimiento similar a la cruza CS155 x V 53 A $(5611 \mathrm{~kg} / \mathrm{ha})$, lo que probablemente se debió a que esta combinación no mostró efecto heterótico (Cuadro 3).

En las variables floración masculina y femenina, altura de planta y mazorca, peso volumétrico, peso de 200 granos y longitud de mazorca, se presentaron diferencias entre las variedades, que probablemente se debe a la limitada humedad presente en el ciclo, condición que propicia expresiones diferentes de los

Cuadro 3. Comparación de medias (Tukey) de genotipos (variedades, cruzas simples e híbridos no convencionales de maíz), para variables evaluadas, considerando la media de tres ambientes de Valles Altos, México. Ciclo primavera-verano 2012.

\begin{tabular}{|c|c|c|c|c|c|c|c|c|}
\hline Genotipos & $\begin{array}{l}\text { Rend. } \\
\text { (kg/ha) }\end{array}$ & $\begin{array}{l}\text { DFM } \\
\text { (días) }\end{array}$ & $\begin{array}{l}\text { DFF } \\
\text { (días) }\end{array}$ & $\begin{array}{c}\mathbf{A P} \\
(\mathbf{c m})\end{array}$ & $\begin{array}{l}\text { AM } \\
(\mathbf{c m})\end{array}$ & $\begin{array}{c}\text { PV } \\
(\mathbf{k g} / \mathbf{h l})\end{array}$ & $\begin{array}{l}\text { P200S } \\
\text { (g) }\end{array}$ & $\begin{array}{l}\mathbf{L M} \\
(\mathbf{c m})\end{array}$ \\
\hline CS156 x V 54 A & 6796 a & $72 \mathrm{ab}$ & $73 \mathrm{de}$ & $227 \mathrm{a}$ & $95 \mathrm{bcd}$ & $779 \mathrm{ab}$ & $60,6 \mathrm{ab}$ & $16,5 \mathrm{abcd}$ \\
\hline CS156 x V55 A & $6204 \mathrm{ab}$ & $73 \mathrm{ab}$ & 74 cde & $228 \mathrm{a}$ & 98 bcd & $769 \mathrm{ab}$ & $63,3 \mathrm{ab}$ & $17,1 \mathrm{ab}$ \\
\hline CS156 x OU2C & $5985 \mathrm{abc}$ & $73 \mathrm{ab}$ & 74 cde & $234 \mathrm{a}$ & $100 \mathrm{bc}$ & $776 \mathrm{ab}$ & $61,7 \mathrm{ab}$ & $17,5 \mathrm{a}$ \\
\hline $\mathrm{V}-53 \mathrm{~A}$ & $5947 \mathrm{abc}$ & $66 \mathrm{~b}$ & $73 \mathrm{de}$ & $225 \mathrm{ab}$ & $114 \mathrm{a}$ & $764 \mathrm{ab}$ & $65,6 \mathrm{a}$ & $14,7 \mathrm{~cd}$ \\
\hline CS155 x V53 A & $5611 \mathrm{abc}$ & $73 \mathrm{ab}$ & $73 \mathrm{de}$ & $223 \mathrm{ab}$ & $100 a b c$ & $760 \mathrm{ab}$ & $64,1 \mathrm{ab}$ & $16,3 \mathrm{abcd}$ \\
\hline CS156 x OP2D & $5530 \mathrm{abc}$ & $71 \mathrm{ab}$ & $72 \mathrm{de}$ & 229 a & $95 \mathrm{bcd}$ & $765 \mathrm{ab}$ & $64,6 \mathrm{a}$ & $16,3 \mathrm{abcd}$ \\
\hline V-55 A & $5288 \mathrm{bcd}$ & $71 \mathrm{ab}$ & $72 \mathrm{de}$ & 229 a & $105 \mathrm{ab}$ & 786 a & $65,6 \mathrm{a}$ & $14,3 \mathrm{~d}$ \\
\hline V-54 A & $4918 \mathrm{bcd}$ & $71 \mathrm{ab}$ & $73 \mathrm{de}$ & $213 \mathrm{abcd}$ & $101 \mathrm{abc}$ & $772 \mathrm{ab}$ & $62,4 \mathrm{ab}$ & $14,7 \mathrm{~cd}$ \\
\hline CS156 x OU3C & $4909 \mathrm{bcd}$ & $73 \mathrm{ab}$ & 74 bcd & $223 \mathrm{ab}$ & 92 bcd & $781 \mathrm{ab}$ & $62,8 \mathrm{ab}$ & $16,8 \mathrm{abc}$ \\
\hline CS155 x V54 A & $4691 \mathrm{~cd}$ & $71 \mathrm{ab}$ & $72 \mathrm{e}$ & $222 a b c$ & 95 bcd & $766 \mathrm{ab}$ & $61,1 \mathrm{ab}$ & 15,7 abcd \\
\hline CS155 & $4190 \quad \mathrm{~d}$ & 75 a & $77 \mathrm{~b}$ & 214 abcd & $90 \mathrm{~cd}$ & $746 \mathrm{~b}$ & $57,3 \mathrm{abc}$ & $16,3 \mathrm{abcd}$ \\
\hline OU3C & $2842 \mathrm{e}$ & $72 \mathrm{ab}$ & $73 \mathrm{de}$ & $198 \mathrm{~cd}$ & $91 \mathrm{~cd}$ & $775 \mathrm{ab}$ & $61,7 \mathrm{ab}$ & $14,9 \mathrm{bcd}$ \\
\hline CS156 & 2764 & $78 \mathrm{a}$ & $81 \quad \mathrm{a}$ & 209 abcd & $89 \mathrm{~cd}$ & $751 \mathrm{ab}$ & $49,1 \mathrm{c}$ & $15,4 \mathrm{abcd}$ \\
\hline OP2D & 2242 & $72 \mathrm{ab}$ & $73 \mathrm{de}$ & $194 \mathrm{~d}$ & $84 \mathrm{~d}$ & $774 \mathrm{ab}$ & $60,4 \mathrm{ab}$ & $14,3 \quad \mathrm{~d}$ \\
\hline OU2C & 2023 & $75 \mathrm{a}$ & $76 \mathrm{bc}$ & $202 \mathrm{bcd}$ & $90 \mathrm{~cd}$ & $771 \mathrm{ab}$ & $55,3 \mathrm{bc}$ & $14,5 \mathrm{~cd}$ \\
\hline D.S.H. $(0,05)$ & 1316 & 8 & 3 & 25 & 14 & 37 & 9,2 & 2,2 \\
\hline
\end{tabular}

CS156: cruza simple 156; CS155: cruza simple 155; OU2C: Oro Ultra 2 Cristalino; OP2D: Oro Plus 2 Dentado; OU3C: Oro Ultra 3 Cristalino; D.S.H.: diferencia significativa honesta (corresponde a la diferencia mínima significativa de la Prueba de Tukey). DFM: días a floración masculina; DFF: días a floración femenina; AP: altura de planta; AM: altura de mazorca; PV: peso volumétrico; P200S: peso de 200 semillas; LM: longitud de mazorca.

Las medias con la misma letra estadísticamente en la misma columna, no tienen diferencia significativa (Tukey al 0,05 de significancia). 
genotipos (Tadeo et al., 2012). La floración masculina y femenina presentada por cada una de las variedades, similar estadísticamente, permitió confirmar la precocidad de las variedades en evaluación (Tadeo et al., 2012; Espinosa et al., 2013).

Las variedades V-54 A y V-55 A fueron recientemente inscritas por el INIFAP ante el Catálogo Nacional de Variedades Vegetales, por lo que se incrementó la semilla certificada por parte de microempresas para el abastecimiento de productores de Valles Altos de México (Espinosa et al., 2008a; Espinosa et al., 2010; Espinosa et al., 2011), como una alternativa de abastecimiento de semillas ante la ausencia de la Productora Nacional de Semillas (Espinosa et al., 2003; Espinosa et al., 2014). Si bien las dos variedades representan una buena opción para los productores de maíz, que siembran bajo condiciones de secano, en fechas tardías, se observó que el rendimiento de la variedad V-54 A (4918 kg/ha), fue superado significativamente por la cruza no convencional $156 \mathrm{x}$ V 54 A (6796 kg/ha), con un porcentaje de $38,1 \%$. En la cruza no convencional, la variedad V-54 A, participó como uno de los progenitores (Cuadro 3). Lo anterior señala que esta cruza no convencional aportaría mayor productividad en siembras comerciales en comparación con la variedad V-54 A, que ya utilizan en forma comercial. A diferencia de lo anterior, la variedad V 53 A rindió $5947 \mathrm{~kg} / \mathrm{ha}$, que representa $105,9 \%$ respecto a la cruza no convencional CS155 X V 53 A (5611 $\mathrm{kg} / \mathrm{ha}$ ), ambos valores son estadísticamente similares, lo que significa que en este caso, el híbrido no convencional no fue superior a la variedad progenitora V 53 A. Con base en lo anterior, se podría deducir que no en todos los casos, los híbridos no convencionales representan ventaja, por lo que conviene evaluar los materiales de este trabajo en otros ciclos para confirmar la productividad de los genotipos y la probable ventaja en algunos casos.

Con respecto a la floración masculina y femenina, el mejor híbrido no convencional (156 x V 54 A), presentó similar número de días a floración masculina (72 días) y femenina (73 días) con respecto a la variedad V 54 A (71 y 73 días respectivamente), lo que es positivo porque mantuvo buena precocidad, que es factor importante para la utilización en fechas de siembra retrasadas, como las requieren las condiciones y los productores de esa región de Valles Altos (2200 a $2600 \mathrm{msnm}$ ). La floración masculina y femenina presentada por cada uno de los otros genotipos, permitió confirmar la precocidad de estos materiales en evaluación.

\section{LITERATURA CITADA}

Ávila, P.M.A., J.L. Arellano, J. Virgen, y A.J. Gámez. 2009. H-52 híbrido de maíz para Valles Altos de La Mesa Central de México. Agric. Téc. Méx. 35:237-240.

Espinosa, A., M.M. Sierra, y M.N. Gómez. 2003. Producción y tecnología de semillas mejoradas de maíz por el INIFAP en el escenario sin la PRONASE. Agron. Mesoam. 14:117-121.

Espinosa, C.A., M. Tadeo, N. Gómez, M. Sierra, R. Martínez, J. Virgen, A. Palafox, F. Caballero, G. Vázquez, y M. Salinas. 2008a. V-53 A variedad mejorada de polinización libre de grano amarillo para Valles Altos de México. En: Memoria Técnica No. 9, Día de Campo: CEVAMEX 2008. INIFAP, Chapingo, México.p. 33-34.

Espinosa, A., M. Tadeo, A. Turrent, N. Gómez, M. Sierra, F. Caballero, R. Valdivia, y F. Rodríguez. 2008b. El potencial de las variedades nativas y mejoradas de maíz. Revista de Difusión de la Facultad de Ciencias de la UNAM 92-93:118-125.

Espinosa, A., M. Tadeo, N. Gómez, M. Sierra, J. Virgen, A. Palafox, F. Caballero, G. Vázquez, F. Rodríguez, R. Valdivia. 2010. V-54 A, nueva variedad de maíz de grano amarillo para siembras de temporal retrasado en los Valles Altos de México. Revista Mexicana de Ciencias Agrícolas 1:677-680.

Espinosa, A., M. Tadeo, N. Gómez, M. Sierra, J. Virgen, A. Palafox, F. Caballero, G. Vázquez, F. Rodríguez, R. Valdivia, I. Arteaga, y R. González. 2011. V-55 A, variedad de maíz de grano amarillo para los Valles Altos de México. Rev. Fitotec. Mex. 34:149-150.

Espinosa, A., M. Tadeo, A. Turrent, M. Sierra, N. Gómez, y B. Zamudio. 2013. Rendimiento de variedades precoces de maíz de grano amarillo para Valles Altos de México. Agron. Mesoam. 24:93-99.

Espinosa, A., A. Turrent, M. Tadeo, A. San Vicente, N. Gómez, R. Valdivia, M. Sierra, y B. Zamudio. 2014. Ley de Semillas y Ley Federal de Variedades Vegetales y transgénicos de maíz en México. Revista Mexicana de Ciencias Agrícolas 5:293-308.

García, E. 2004. Modificaciones al sistema de clasificación climática de Köppen. Instituto Nacional de Geografía. Universidad Nacional Autónoma de México. 
SAS Institute. 1996. SAS Users' guide: Statistics, Version 6. SAS Inst., Cary, NC, USA.

Tadeo, M., y C. Espinosa. 2004. Producción de semilla y difusión de variedades e híbridos de maíz de grano amarillo para Valles Altos de México. Revista FESC Divulgación Científica Multidisciplinaria 4(14):5-10.

Tadeo, M., A. Espinosa, R. Valdivia, N. Gómez, M. Sierra, y B. Zamudio. 2010. Vigor de las semillas y productividad de variedades de maíz. Agron. Mesoam. 21:31-38.
Tadeo, M., A. Espinosa, I. Arteaga, V. Trejo, M. Sierra, R. Valdivia,y B.Zamudio. 2012. Productividad de variedades precoces de maíz de grano amarillo para Valles Altos. Revista Mexicana de Ciencias Agrícolas 3:1417-1423.

Turrent, A. 1994. Plan de investigación del Sistema maíztortilla en la región Centro. CIRCE, INIFAP, SARH, Publicación especial Núm. 12, Chapingo, México. 55 p. Turrent, F.A. 2009. El potencial productivo del maíz. Revista de Difusión de la Facultad de Ciencias de la UNAM 92-93:126-129. 